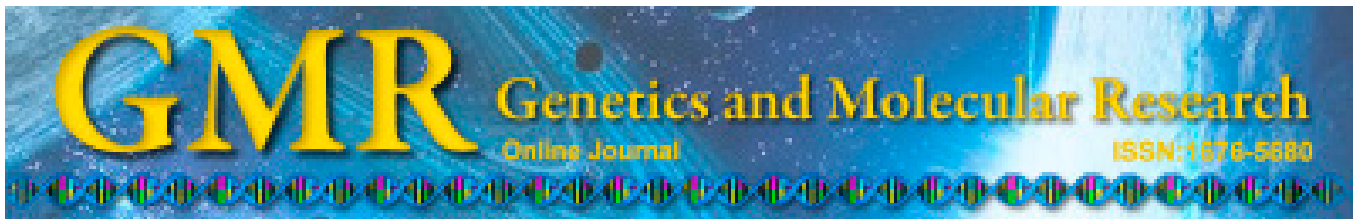

\title{
Clinical significance of SHMT1 rs1979277 polymorphism in Asian solid tumors: evidence from a meta-analysis
}

\author{
T.T. Zhao ${ }^{1 *}$, L.L. Shen ${ }^{1 *}$, X.L. Zhang ${ }^{2 *}$, D.Y. Gu ${ }^{1}$, Q. Zhang ${ }^{1}$, X.Y. Huo ${ }^{1}$, \\ C.J. Tang ${ }^{1}$ and J.F. Chen ${ }^{1}$ \\ ${ }^{1}$ Department of Oncology, Nanjing First Hospital, Nanjing Medical University, \\ Nanjing, China \\ ${ }^{2}$ Department of Oncology, Nantong Tumor Hospital, Nantong, China \\ *These authors contributed equally to this study. \\ Corresponding author: J.F. Chen \\ E-mail: jinfeichen@sohu.com
}

Genet. Mol. Res. 14 (2): 5602-5614 (2015)

Received July 31, 2014

Accepted January 19, 2015

Published May 25, 2015

DOI http://dx.doi.org/10.4238/2015.May.25.12

\begin{abstract}
Published data regarding the association between the cytosolic serine hydroxymethyltransferase (SHMT1) C1420T (Leu474Phe) polymorphism and solid tumor risk have shown inconclusive results. To derive a more precise estimation of the relationship, we performed a meta-analysis of 23 published studies that included 14,409 cancer cases and 16,996 controls. A comprehensive search was conducted to identify all eligible studies of the SHMT1 rs 1979277 polymorphism and solid tumor risk. The pooled odds ratios (ORs) and the $95 \%$ confidence intervals $(95 \% \mathrm{CIs})$ were calculated using a fixed- or random-effects model. Heterogeneity was represented by $P_{H}$; publication bias and sensitivity analysis were also explored. Overall, no significant associations were found for any genetic models tested. However, upon stratification by cancer type, a significant decreased risk of breast cancer risk was identified in the homozygote comparison (OR $=0.79,95 \% \mathrm{CI}=0.65-0.97$ for $\mathrm{TT}$ versus $\mathrm{CC})$. An analysis stratified
\end{abstract}


by ethnicity and source of controls revealed an obvious decrease in risk among Asian groups in all genetic models, and among populationbased controls only in the homozygote comparison and recessive model. Therefore, our meta-analysis suggested that the SHMT1 C1420T polymorphism was associated with decreased risk of breast cancer. Significant protective effects were found among Asian populations, but not in Caucasian groups. Due to some minor limitations, our findings should be confirmed by further studies.

Key words: Cancer; Single nucleotide polymorphism; Meta-analysis; Cytosolic serine hydroxymethyltransferase C1420T

\section{INTRODUCTION}

Cancer is a multistage progressive disease with high morbidity and mortality worldwide (Pharoah et al., 2004). In 2013, a total of 1,665,540 new cancer cases and 585,720 cancer deaths occurred in the United States. Cancers of the lung and bronchus, prostate, colorectum, and breast continue to be the most common causes of cancer deaths, which have become a worldwide public health burden (Siegel et al., 2014). Cancer is a multistep, multi-factorial disease that involves a complex interplay between genetic and environmental factors. However, the complex etiology of cancer is not fully elucidated. The continually increasing incidence and mortality rates lead researchers to speculate that dietary, infectious, cultural, environmental, and/or genetic factors might be implicated in the etiology of cancer (Foulkes, 2008).

Genetic polymorphisms and dietary micronutrients of one-carbon metabolism were studied extensively in different cancers, as these factors influence DNA synthesis, repair, and methylation. The principal mechanisms of carcinogenesis by these factors are uracil misincorporation into DNA causing DNA damage (Melnyk et al., 1999), or aberrant methylation (focal hypermethylation and global hypomethylation) that triggers the inactivation of tumor suppressor genes and inactivation of proto-oncogenes (Christman et al., 1993). One-carbon metabolism harbors several crucial biological reactions from folate uptake to synthesis of S-adenosylmethionine (SAM), which involve several enzymes in the folate biological network, including methylenetetrahydrofolate reductase, thymidylate synthase, methyltetrahydrofolatehomocysteine methyltransferase, cytosolic serine hydroxymethyltransferase (cSHMT) SHMT1), reduced folate carrier 1, glutamate carboxypeptidase II, and methyltetrahydrofolatehomocysteine methyltransferase reductase, among others. Any perturbation in this metabolic pathway can impair the synthesis of SAM, a universal methyl group donor, thereby leading to epigenetic changes, specifically aberrant DNA methylation (Naushad et al., 2011). Recently, many studies have been conducted to investigate the associations between the SHMT1 C1420T polymorphism and diverse cancer risks in different countries. However, the results from these studies remain inconclusive. Therefore, our meta-analysis focused on SHMT1 C1420T rather than other enzymes.

SHMT1, located on chromosome 17p11.2, encodes a vitamin B6-dependent enzyme that catalyzes the reversible transfer of the hydroxymethyl group of serine to tetrahydrofolate to form 5,10-methylene tetrahydrofolate and glycine and the irreversible conversion of 5,10-methylene tetrahydrofolate to 5-formyl tetrahydrofolate (futile folate cycle). Formation of 5-formyl tetrahydrofolate helps in maintaining one-carbon homeostasis during the rapidly 
proliferative stages of development (Fu et al., 2005; Niclot et al., 2006). Rs1979277(C > T) is a non-synonymous single nucleotide polymorphism (SNP) located in the 12th exon of the SHMT1 gene (C1420T) and results in an amino acid change from leucine (Leu) to phenylalanine (Phe) at codon 474 of SHMT1. This common polymorphism results in reduced plasma and red blood cell folate levels in carriers of the 1420CC genotype (Heil et al., 2001), and could mimic a folate deficiency by reducing the one-carbon moieties available for both remethylation of homocysteine and DNA synthesis.

To date, a number of studies have investigated the role of the SHMT1 C1420T polymorphism in the etiology of cancers of various organs, including lung, bladder, colorectal, breast, prostate, and others. However, the results of these studies remain inconclusive. In consideration of the extensive role of SHMT1 in the carcinogenic process, we carried out a metaanalysis on all eligible case-control studies to estimate the overall cancer risk of the SHMTI C1420T polymorphism and to quantify the potential between-study heterogeneity.

\section{MATERIAL AND METHODS}

\section{Identification and eligibility of relevant studies}

To identify relevant studies for the performance of a meta-analysis, PubMed, Embase, and Medline were searched using the search terms: "rs1979277", "SHMT1", "cSHMT", "SNP", "polymorphism or variation", and "carcinoma or cancer or neoplasm or tumour or tumor" (last search updated on February 12, 2014). The search was limited to English language papers. We also used the PubMed option "Related Articles" in each research article to search for further potentially relevant articles. Additional studies were identified by a manual search of the references of the original studies. In the case of studies with overlapping published data, we selected the most recent study that included a large number of subjects. All the studies we explored provided ethics statements and statements of informed consent. Studies included in our meta-analysis were required to meet the following criteria: a) evaluation of the C1420T polymorphism and cancer risk; b) use of a case-control design; and c) contain available genotype frequencies.

\section{Data extraction}

Two investigators independently extracted data and reached a consensus on all items. For each study, the following data were considered: the first author's last name, year of publication, country of origin, ethnicity, cancer type, genotyping method, value of Hardy-Weinberg equilibrium, numbers of genotyped cases and controls, and source of control groups (population- or hospital-based controls). Different ethnic descents were categorized as Caucasian or Asian. For studies including subjects of different ethnic groups, data were extracted separately for each ethnic group whenever possible.

\section{Statistical analysis}

The strength of the association between the SHMT rs1979277 polymorphism and cancer risk was assessed by the odds ratio (OR) and the $95 \%$ confidence interval $(95 \% \mathrm{CI})$. Pooled ORs were obtained from the combination of individual studies by heterozygote (CT 
$v_{s} \mathrm{CC}$ ) or homozygote comparison (TT vs $\left.\mathrm{CC}\right)$, or a dominant (CT/TT $\left.v_{s} \mathrm{CC}\right)$ or a recessive model (TT vs CT/CC). The significance of pooled ORs was determined using the Z-test. Both the Cochran's Q statistic to test for heterogeneity and the $\mathrm{I}^{2}$ statistic to quantify the proportion of the total variation due to heterogeneity were calculated to estimate heterogeneity among the studies included (Cochran, 1950; Higgins et al., 2003). If the $P_{H}$ value of the $\mathrm{Q}$ test was $<0.05$, indicating a lack of heterogeneity across studies, the summary OR estimate of each study was calculated by the fixed-effects model (the Mantel-Haenszel method); otherwise, the random-effects model (the DerSimonian and Laird method) was used. Stratified analyses were also performed by ethnicity, cancer type (if one cancer type contained less than two individual studies, it was combined into the "other cancers" group), and source of controls. Sensitivity analyses were performed to evaluate the stability of the results by sequentially deleting a single study in the meta-analysis to show the influence of the individual data set to the pooled OR. Funnel plots and Egger's linear regression tests were used to assess the potential publication bias (Egger et al., 1997). All analyses were performed using the Statistics/Data Analysis software (version10.0; StataCorp LP, College Station, TX, USA), using two-sided P values.

\section{RESULTS}

\section{Study characteristics}

Over 139 published articles relevant to the search terms were identified (Figure 1); 116 were excluded based on clinical data quality checks. Through screening of the titles, 75 articles were excluded (18 were not on the topic of solid tumor research, 23 were not performed in humans, and 34 did not examine polymorphisms). Abstracts from 64 articles were reviewed, and an additional 27 studies were excluded, leaving 37 studies for full publication review. Of these, 11 additional articles were excluded (five were reviews, and six were not case-control studies). Three articles were excluded because of a lack of usable data. Eventually, a total of 23 eligible studies involving 14,409 cases and 16,996 controls were included in the pooled analyses (Chen et al., 2004; Zhang et al., 2005; Koushik et al., 2006; Hazra et al., 2007; Lissowska et al., 2007; Moore et al., 2007; van den Donk et al., 2007; Wang et al., 2007; Xu et al., 2007; Yu et al., 2007; Guerreiro et al., 2008; Steck et al., 2008; Stevens et al., 2008; Bentley et al., 2010; Komlosi et al., 2010; Vainer et al., 2010; Curtin et al., 2011; Mohammad et al., 2011; Naushad et al., 2011, 2012; Weiner et al., 2012; Succi et al., 2014; Wu et al., 2014). The characteristics of the studies selected are summarized in Table 1. All studies were case-control studies, including nine breast cancer, eight colorectal cancer, two head and neck cancer, and two prostate cancer studies, and one lung cancer and one bladder cancer study. There were five studies on Asians and 18 studies on Caucasians. Cancers were confirmed histologically or pathologically in most studies. The TaqMan assay was utilized in 11 of the 23 studies. In addition, controls were primarily matched by gender and age; in 11 studies, the source of controls was population-based, and hospital-based controls were used in the remaining 12 studies. The distributions of genotypes in the controls were consistent with Hardy-Weinberg equilibrium except for six studies (Yu et al., 2007; Guerreiro et al., 2008; Steck et al., 2008; Komlosi et al., 2010; Curtin et al., 2011; Naushad et al., 2012), which were tested in the sensitivity analyses. 


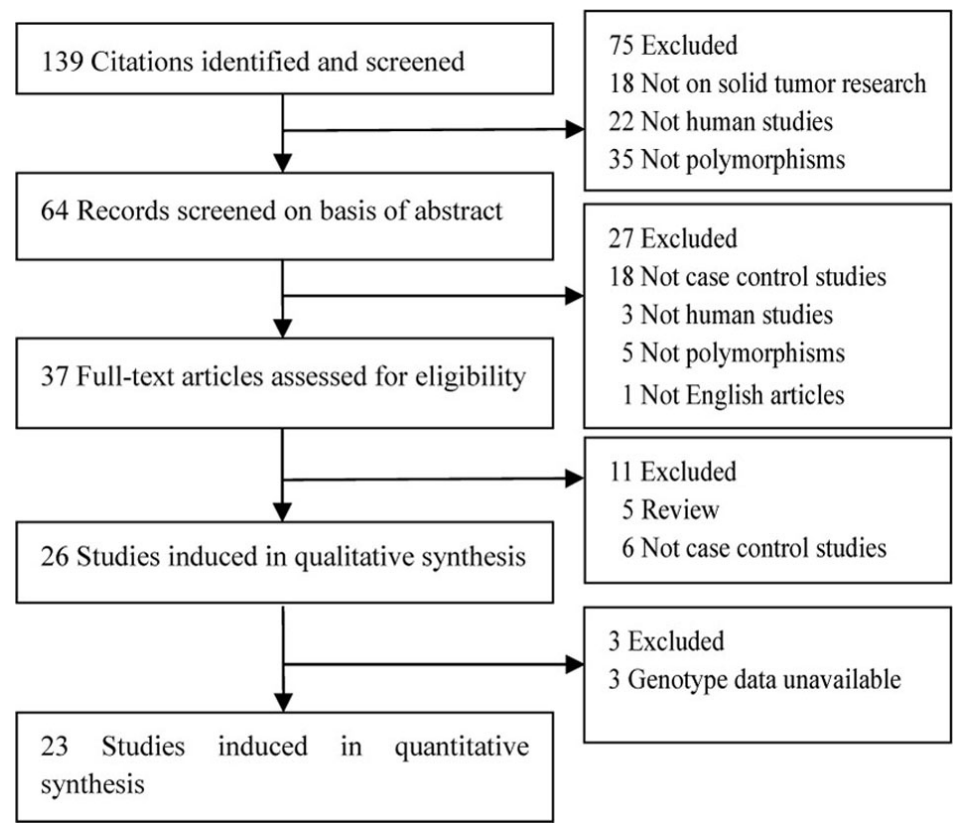

Figure 1. Flow diagram summarizing the search strategy.

Table 1. Characteristics of literature included in the meta-analysis.

\begin{tabular}{|c|c|c|c|c|c|c|c|c|c|c|c|c|c|}
\hline \multirow[t]{2}{*}{ First author } & \multirow[t]{2}{*}{ Year } & \multirow[t]{2}{*}{ Country } & \multirow[t]{2}{*}{ Ethnicity } & \multirow{2}{*}{$\begin{array}{l}\text { Source of } \\
\text { controls }\end{array}$} & \multirow{2}{*}{$\begin{array}{l}\text { Cancer } \\
\text { type }\end{array}$} & \multirow{2}{*}{$\begin{array}{l}\text { Genotyping } \\
\text { method }\end{array}$} & \multicolumn{3}{|c|}{ Patients (N) } & \multicolumn{3}{|c|}{ Controls (N) } & \multirow[t]{2}{*}{ HWE } \\
\hline & & & & & & & $\mathrm{CC}$ & $\mathrm{CT}$ & TT & $\mathrm{CC}$ & $\mathrm{CT}$ & TT & \\
\hline issowska & 2007 & Poland & Caucasian & PB & $\mathrm{BC}$ & Taqman & 819 & 919 & 222 & 965 & 1040 & 252 & 0.26 \\
\hline Mohammd & 2011 & India & Asian & HB & $\mathrm{BC}$ & PCR-RFLP & 53 & 116 & 53 & 43 & 113 & 79 & 0.82 \\
\hline Naushad & 2012 & India & Asian & PB & $\mathrm{BC}$ & PCR-RFLP & 52 & 144 & 92 & 24 & 154 & 75 & $2 \mathrm{E}-05$ \\
\hline Naushad & 2011 & India & Asian & PB & $\mathrm{BC}$ & PCR-RFLP & 56 & 128 & 60 & 43 & 119 & 82 & 0.99 \\
\hline Bentley & 2010 & US & Caucasian & $\mathrm{HB}$ & $\mathrm{BC}$ & TaqMan & 463 & 386 & 90 & 619 & 489 & 118 & 0.14 \\
\hline $\mathrm{Wu}$ & 2014 & China & Asian & HB & $\mathrm{BC}$ & PCR-RFLP & 48 & 39 & 9 & 32 & 36 & 17 & 0.25 \\
\hline $\mathrm{Yu}$ & 2007 & Taiwan & Asian & PB & $\mathrm{BC}$ & PCR-RFLP & 79 & 20 & 6 & 283 & 89 & 31 & $3 \mathrm{E}-08$ \\
\hline $\mathrm{Xu}$ & 2007 & US & Caucasian & PB & $\mathrm{BC}$ & PCR-RFLP & 508 & 459 & 94 & 505 & 477 & 117 & 0.78 \\
\hline Vainer & 2010 & Russia & Caucasian & PB & $\mathrm{BC}$ & TaqMan & 387 & 349 & 94 & 367 & 357 & 85 & 0.89 \\
\hline Succi & 2014 & Brazil & Caucasian & PB & $\mathrm{HNC}$ & TaqMan & 124 & 95 & 18 & 260 & 183 & 45 & 0.13 \\
\hline Zhang & 2005 & US (Houston) & Caucasian & HB & $\mathrm{HNC}$ & PCR-RFLP & 330 & 294 & 97 & 575 & 522 & 137 & 0.26 \\
\hline Wang & 2007 & US (Houston) & Caucasian & HB & $\mathrm{LC}$ & PCR-RFLP & 459 & 431 & 142 & 495 & 504 & 146 & 0.32 \\
\hline Curtin & 2011 & US & Caucasian & PB & CRC & Illumina $^{\mathrm{TM}}$ & 362 & 287 & 77 & 455 & 363 & 110 & 0.005 \\
\hline Koushik & 2006 & US & Caucasian & HB & $\mathrm{CRC}$ & TaqMan & 172 & 141 & 37 & 370 & 358 & 75 & 0.38 \\
\hline Chen & 2004 & US & Caucasian & PB & $\mathrm{CRC}$ & PCR-RFLP & 131 & 110 & 28 & 221 & 196 & 41 & 0.79 \\
\hline Hazra & 2007 & US & Caucasian & HB & $\mathrm{CRC}$ & Taqman & 235 & 243 & 43 & 237 & 233 & 49 & 0.45 \\
\hline van den Donk & k 2007 & Netherlands & Caucasian & HB & $\mathrm{CRC}$ & RT-PCR & 361 & 318 & 64 & 336 & 293 & 70 & 0.6 \\
\hline Steck & 2008 & US & Caucasian & PB & CRC & TaqMan & 151 & 128 & 28 & 257 & 205 & 71 & 0.004 \\
\hline Guerreiro & 2008 & Portu & Caucasian & HB & $\mathrm{CRC}$ & TaqMan & 94 & 76 & 26 & 84 & 107 & 9 & $5 \mathrm{E}-04$ \\
\hline Komlosi & 2010 & Hungary & Caucasian & PB & $\mathrm{CRC}$ & PCR-RFLP & 477 & 393 & 85 & 440 & 384 & 115 & 0.03 \\
\hline Moore & 2007 & Spain & Caucasian & HB & $\mathrm{BDC}$ & TaqMan & 590 & 426 & 76 & 538 & 400 & 73 & 0.91 \\
\hline Stevens & 2008 & US & Caucasian & HB & $\mathrm{PC}$ & TaqMan & 558 & 470 & 116 & 576 & 461 & 107 & 0.29 \\
\hline Weiner & 2012 & Russia & Caucasian & $\mathrm{HB}$ & $\mathrm{PC}$ & TaqMan & 164 & 155 & 52 & 133 & 124 & 27 & 0.81 \\
\hline
\end{tabular}

$\mathrm{BC}=$ breast cancer; $\mathrm{HNC}=$ head and neck (oral cavity, pharynx, and larynx) cancer; $\mathrm{CRC}=$ colorectal cancer; $\mathrm{LC}=$ lung cancer; $\mathrm{PC}=$ prostate cancer; $\mathrm{BDC}=$ bladder cancer; $\mathrm{PB}=$ population-based; $\mathrm{HB}=$ hospital-based; HWE $=$ Hardy-Weinberg equilibrium; RFLP $=$ restriction fragment length polymorphism; PCR $=$ polymerase chain reaction; Illumina ${ }^{\mathrm{TM}}=$ Illumina $^{\mathrm{TM}}$ Golden Gate bead-based genotyping platform. 


\section{Quantitative synthesis}

Overall, as shown in Table 2, no significant associations were found for any genetic model examined (homozygote comparison, TT versus CC: $\mathrm{OR}=0.92,95 \% \mathrm{CI}=$ $0.82-1.03, P_{H}=0.008, \mathrm{I}^{2}=46.3 \%$; heterozygote comparison, $\mathrm{TC}$ versus $\mathrm{CC}$ : $\mathrm{OR}=0.97$, $95 \% \mathrm{CI}=0.93-1.02, P_{H}=0.528, \mathrm{I}^{2}=0.0 \%$; dominant model, CT/TT versus $\mathrm{CC}: \mathrm{OR}=$ $0.97,95 \% \mathrm{CI}=0.93-1.01, P_{H}=0.461, \mathrm{I}^{2}=0.0 \%$; recessive model, $\mathrm{TT}$ versus $\mathrm{CT} / \mathrm{CC}: \mathrm{OR}$ $\left.=0.95,95 \% \mathrm{CI}=0.85-1.06, P_{H}=0.005, \mathrm{I}^{2}=48.9 \%\right)$. However, in the subgroup analysis by ethnicity, statistically significantly protective effects were found among Asians $(\mathrm{OR}=$ $0.55,95 \% \mathrm{CI}=0.41-0.72, P_{H}=0.886, \mathrm{I}^{2}=0.0 \%$ for $\mathrm{TT}$ versus $\mathrm{CC} ; \mathrm{OR}=0.71,95 \% \mathrm{CI}=$ $0.56-0.90, P_{H}=0.359, \mathrm{I}^{2}=8.4 \%$ for $\mathrm{CT}$ versus $\mathrm{CC} ; \mathrm{OR}=0.66,95 \% \mathrm{CI}=0.53-0.82, P_{H}=$ $0.68, \mathrm{I}^{2}=0.0 \%$ for TT/CT versus CC; $\mathrm{OR}=0.75,95 \% \mathrm{CI}=0.61-0.92, P_{H}=0.103, \mathrm{I}^{2}=48 \%$ for TT versus $\mathrm{CC} / \mathrm{CT}$ ). Following stratification by cancer type, there was a significantly decreased risk of breast cancer $\left(\mathrm{OR}=0.79,95 \% \mathrm{CI}=0.65-0.97, P_{H}=0.027, \mathrm{I}^{2}=53.8 \%\right.$ for TT versus $\mathrm{CC}$ ). In the stratified analysis by source of controls, studies with populationbased controls also showed negative associations with cancer risk in two genetic comparisons (homozygote comparison, TT $v s \mathrm{CC}$ : $\mathrm{OR}=0.86,95 \% \mathrm{CI}=0.77-0.96, P_{H}=0.163, \mathrm{I}^{2}$ $=29.6 \%$; and the recessive model, TT $v s \mathrm{CT} / \mathrm{CC}: \mathrm{OR}=0.89,95 \% \mathrm{CI}=0.81-0.987, P_{H}=$ $\left.0.193, \mathrm{I}^{2}=26.3 \%\right)$.

\section{Test of heterogeneity}

When evaluating the association between the SHMT1 rs1979277 polymorphism and the susceptibility to cancer, we found that there was significant heterogeneity for the homozygote comparison (TT $v_{S} \mathrm{CC}: P_{H}=0.008, \mathrm{I}^{2}=46.3 \%$ ) and the recessive model comparison (TT $v s \mathrm{CT} / \mathrm{CC}: P_{H}=0.005, \mathrm{I}^{2}=48.9 \%$ ), but not for the heterozygote comparison (GA $v s$ AA: $\left.P_{H}=0.528, \mathrm{I}^{2}=0.0 \%\right)$ and the dominant model $\left(\mathrm{CT} / \mathrm{TT}\right.$ versus CC: $\left.P_{H}=0.461, \mathrm{I}^{2}=0.0 \%\right)$. Thus, we assessed the source of heterogeneity for the homozygote comparison by examining cancer type, ethnicity, source of controls, and genotyping method. Meta-regression analyses showed that the ethnicity $(\mathrm{P}<0.001)$ and source of controls $(\mathrm{P}=0.038)$, but not genotyping method $(\mathrm{P}=0.086)$ or site of cancer $(\mathrm{P}=0.09)$, were found to contribute to substantial heterogeneity.

\section{Sensitivity analysis}

In order to reflect the influence of the individual dataset on the pooled ORs, we sequentially deleted a single study involved in the meta-analysis during successive iterations, but the corresponding pooled ORs were not altered significantly (data not shown). Although the genotype distributions in six studies did not follow Hardy-Weinberg equilibrium, the corresponding pooled ORs were not materially altered by including these studies, suggesting that the results of this meta-analysis were stable. 
T.T. Zhao et al.

5608

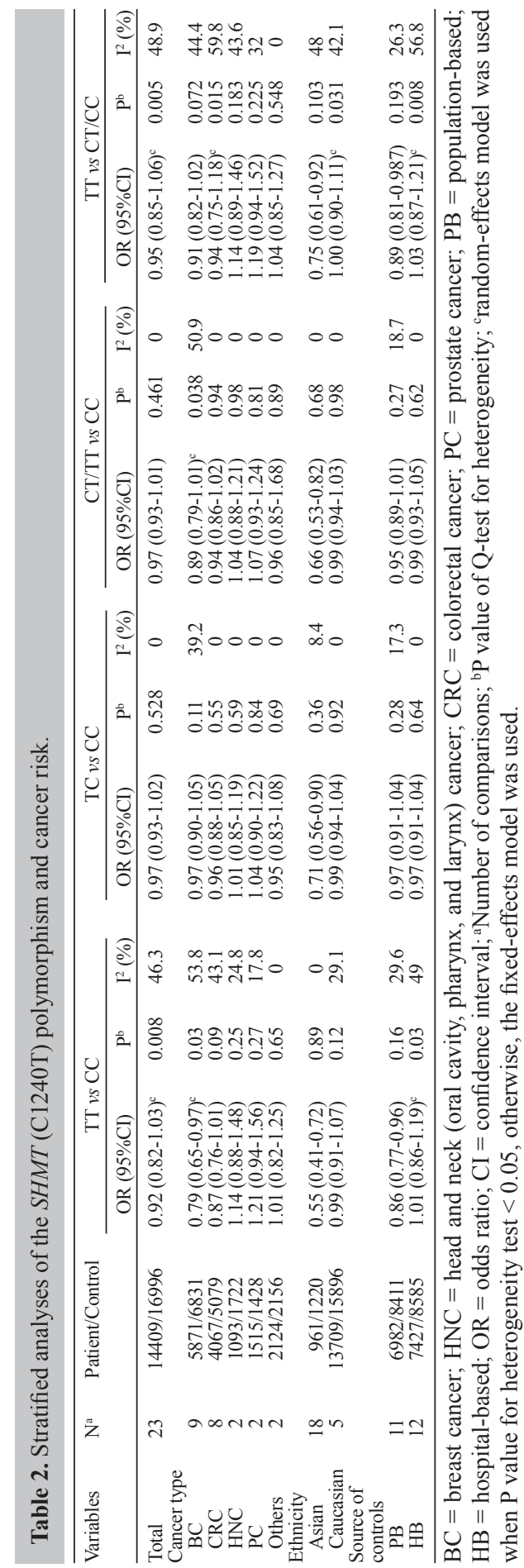

Genetics and Molecular Research 14 (2): $5602-5614$ (2015)

CFUNPEC-RP www.funpecrp.com.br 


\section{Publication bias}

Begg's funnel plot and the Egger test were performed to assess the publication bias of the literature. As shown in Figure 2, the shape of the funnel plots seemed asymmetrical in the heterozygote comparison, suggesting the presence of publication bias. Accordingly, the Egger's test was adopted to provide statistical evidence of funnel plot asymmetry. As expected, the results showed obvious evidence of publication bias $\left(P_{H}=0.002\right.$ for TC versus CC; $P_{H}<0.001$ for TT/TC versus CC). To adjust for this bias, a trim-and-fill method developed by Duval and Tweedie (2000) was implemented. Meta-analysis with or without the trim-and-fill method did not draw different conclusions, indicating that our results were statistically robust.

A

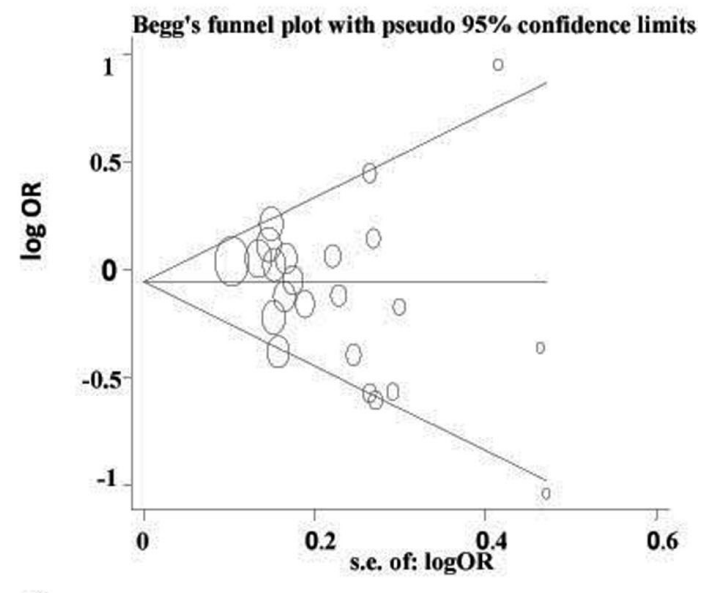

B

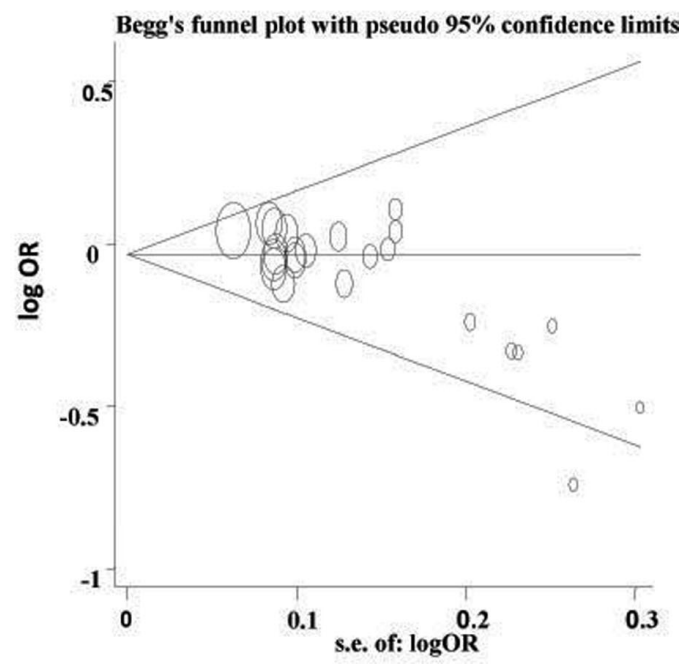

Figure 2. Begg's funnel plots of publication bias tests. A. TT vs CC; B. CT/TT vs CC. Each point represents a separate study for the indicated association. $\log (\mathrm{OR})=$ natural logarithm of OR; horizontal line = mean effect size. 


\section{DISCUSSION}

In the current meta-analysis, we ascertained that the SHMT1 C1420T polymorphism was significantly associated with solid tumor risk, especially with breast cancer. To our knowledge, this is the first study to investigate the association between the SHMT1 C1420T polymorphism and the risk of solid tumors across different ancestries. Although numerous epidemiological studies have demonstrated the effects of SHMT1 C1420T polymorphism on the risk of cancer, the results were conflicting and inconclusive, being based on different cancer sites and small sample sizes in some studies, as shown in Figure 3.

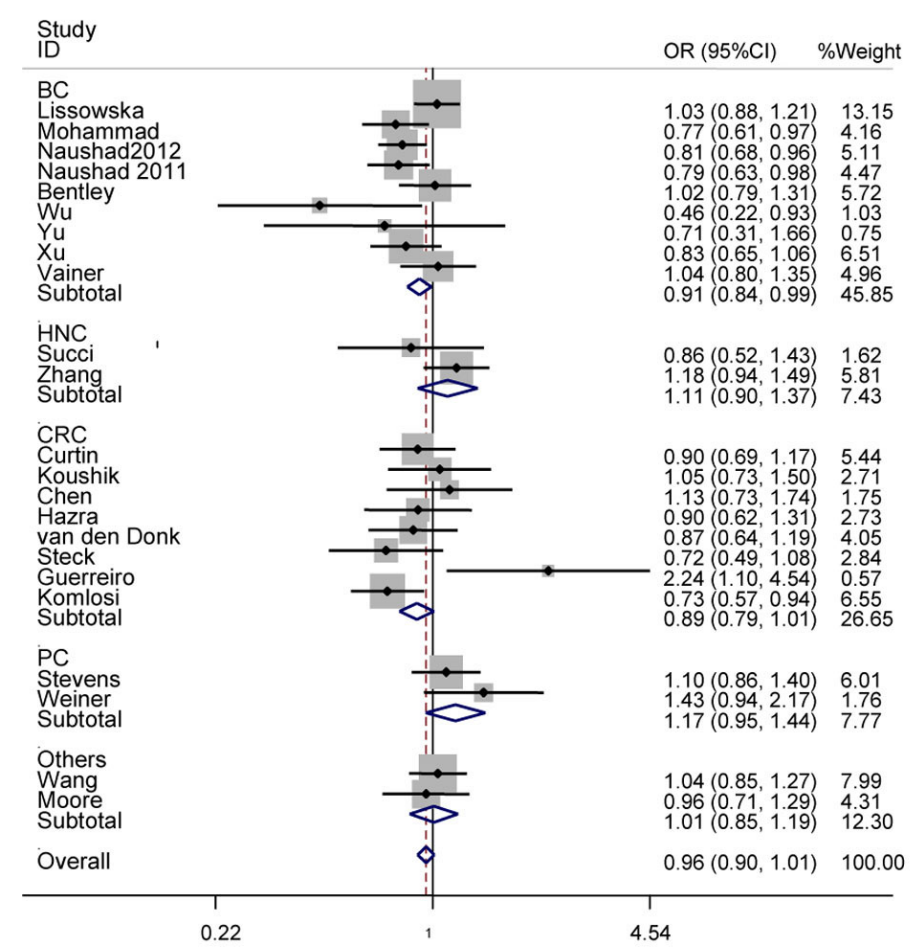

Figure 3. Forest plot from the meta-analysis of $S H M T$ rs1979277 C > T polymorphism and the risk of cancer stratified by cancer type using the homozygote model. SHMT = serine hydroxymethyltransferase; $\mathrm{BC}=$ breast cancer; $\mathrm{HNC}=$ head and neck (oral cavity, pharynx, and larynx) cancer; $\mathrm{CRC}=$ colorectal cancer; $\mathrm{PC}=$ prostate cancer; $\mathrm{CI}=$ confidence interval; $\mathrm{OR}=$ odds ratio.

Polymorphisms in critical enzymes involved in the one-carbon metabolism pathway can influence DNA synthesis, repair and methylation, which play a critical role in carcinogenesis (Lissowska et al., 2007). SHMT1 carries out reversible conversion of tetrahydrofolate to 5,10-methylene tetrahydrofolate (by accepting one-carbon from serine), and irreversible conversion of 5,10-methylene tetrahydrofolate to 5-formyl tetrahydrofolate (futile folate cycle). Formation of 5-formyl tetrahydrofolate helps in maintaining one-carbon homeostasis during the rapidly proliferative stages of development (Fu et al., 2005). The principal mechanisms proposed for the $\mathrm{cSHMT}$ gene variant $(1420 \mathrm{C} \rightarrow \mathrm{T})$ are uracil misincorporation in DNA caus- 
ing DNA damage (Melnyk et al., 1999) and aberrant methylation (focal hypermethylation and global hypomethylation) that triggers the inactivation of tumor suppressor genes and inactivation of proto-oncogenes (Christman et al., 1993).

To date, many studies have been conducted to investigate the association between the SHMT1 C1420T polymorphism and breast cancer risk in different countries. Several studies indicated that there was no association between the SHMT1 C1420T polymorphism and breast cancer risk (Lissowska et al., 2007; Xu et al., 2007; Yu et al., 2007; Bentley et al., 2010; Vainer et al., 2010; Naushad et al., 2012; Wu et al., 2014). However, Naushad et al. (2011) and Mohammad et al. (2011) proved that rs 1979277 polymorphism reduces the risk of breast cancer, which were similar with our result. Estrogens are central in breast tumorigenesis, thus it is possible that polymorphisms in folate metabolizing genes might interact with estrogen metabolism to determine breast cancer risk.(Clemons and Goss, 2001). Estrogens initiate carcinogenesis via metabolic activation to potentially carcinogenic catechol estrogen metabolites (Yager, 2000). The principal pathway for inactivation of catechol estrogen is O-methylation by catechol-O-methyltransferase (COMT) (Cheng et al., 2005; Yager and Liehr, 1996). And SAM is the necessary methyl donor for the COMT-catalyzed reaction. The folate metabolic pathway, which may be negatively impacted by the SHMT1 polymorphic variant, largely determines the SAM level.

Steck et al. (2008) and Komlosi et al. (2010) confirmed that rs1979277 was associated with a significantly decreased risk of colorectal cancer in Netherlands and Hungary, respectively. However, Guerreiro et al. (2008) proved that rs 1979277 increased the risk of colorectal cancer in a Portuguese population. Statistical heterogeneity of these results may correlate with geographical and clinical differences between the studies. Geographical distances between Netherlands, Hungary, and Portugal may reflect the lifestyle and physiological differences of the subjects. In addition, the studies independently focused on differing cancer sites (rectum and colon), contributing to clinical heterogeneity. Physiological effects of folate metabolism have been demonstrated to differ among colorectal cancer sites (Komlosi et al., 2010). However, in our current meta-analysis, no significant associations between the SHMT1 C1420T polymorphism and colorectal cancer risk were observed in any of the genetic models. The null result may be due to the limited number of studies with available data on these characteristics, which had insufficient statistical power to detect a slight effect or which may have generated a fluctuated risk estimate.

When compared by ethnicity, statistically significant increased risks were found among Asians for all genetic comparison but not among Caucasians (Figure 4). Although the exact mechanisms for these ethnic differences are still unknown, possible reasons might be the differences in genetic backgrounds and in the environmental and lifestyle contexts (such as dietary habits, alcohol consumption, and exposure to tobacco smoke) (Hirschhorn et al., 2002). In addition, because of gene-gene interactions, the influence of the SHMT1 rs179277 C $>\mathrm{T}$ polymorphism might be masked or magnified by the presence of other genes that are asof-yet unidentified in the development of cancer. Other factors such as selection bias, different matching criteria, and limited number of studies with available data may yield insufficient statistical power to detect a slight difference and may also generate a fluctuated risk estimate.

We also observed a significantly decreased risk among studies using population-based controls in homozygote comparison and a recessive model, but not using hospital-based controls. Some biases may exist in hospital-based studies, as such controls may represent a sample 
of an ill-defined reference population instead of the general population, particularly when the genotypes investigated were associated with the disease that the hospital-based controls may have. Thus, a proper and representative cancer-free control subject group is very important in reducing biases in such genotype association studies.

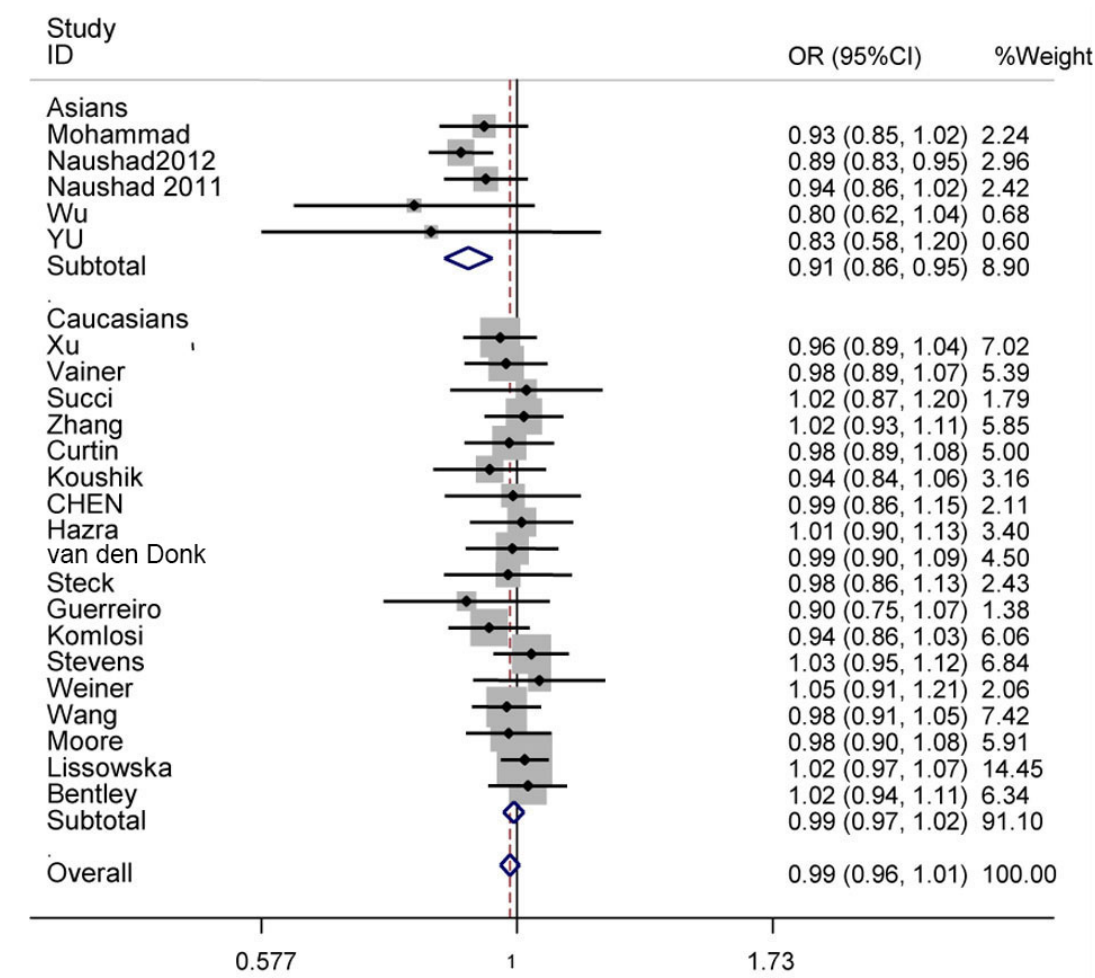

Figure 4. Forest plot from the meta-analysis of $S H M T$ rs $1979277 \mathrm{C}>\mathrm{T}$ polymorphism and the risk of cancer stratified by ethnicity using the dominant model. SHMT $=$ serine hydroxymethyltransferase; $\mathrm{CI}=$ confidence interval; $\mathrm{OR}=$ odds ratio.

Some limitations of this meta-analysis may have affected the objectivity of the conclusions and should be considered in interpreting the results. First, the quantity of published studies was not sufficiently large for a comprehensive analysis, and the lack of original data in some studies limited our further evaluation of potential interactions, such as degree of differentiation, anatomic subsite, and pathological subtype of cancer. Second, although an ideal searching strategy was used to identify eligible studies for the current meta-analysis, it was still possible that a few studies meeting inclusion criteria were not included. Third, the overall outcomes were based on unadjusted estimates, while a more precise evaluation should be conducted if more detailed individual data were available, such as age, gender, histological types, folate intake, and plasma homocysteine levels. The lack of this information might cause serious confounding bias.

In spite of these limitations, our present meta-analysis also had some advantages. First, we estimated the association conclusively between the SHMT1 C > T polymorphism and cancer risk, and further showed a significant association, especially among Asians as op- 
posed to Caucasians. This study might also provide a potential genetic marker and new insight into the etiology of cancer. Second, we pooled a substantial number of patients and controls from different studies, which greatly increased the statistical power of the analysis. Third, the quality of case-control studies included in current meta-analysis was satisfactory and met our inclusion criteria.

In conclusion, our meta-analysis suggests that the SHMT1 C1420T polymorphism decreases the risk of breast cancer. Significant protective effects were found among Asian, but not Caucasian, populations. We suggest that more clinical studies including larger samples stratified by genetic-environmental interaction need to be performed to fully clarify the roles of the SHMT1 polymorphisms in the etiology of cancer.

\section{ACKNOWLEDGMENTS}

Research supported by grants from the National Natural Science Foundation of China (Grant \#81272469), the National "973” Basic Research Program of China (Grant \#2013CB911300), and the Clinical Special Project for the Natural Science Foundation of Jiangsu Province (Grant \#BL2012016); an additional grant comes from a Nanjing 12th FiveYear Key Scientific Project of Medicine to Dr. Jinfei Chen.

\section{REFERENCES}

Bentley AR, Raiszadeh F, Stover PJ, Hunter DJ, et al. (2010). No association between cSHMT genotypes and the risk of breast cancer in the Nurses' Health study. Eur. J. Clin. Nutr. 64: 108-110.

Chen J, Kyte C, Valcin M, Chan W, et al. (2004). Polymorphisms in the one-carbon metabolic pathway, plasma folate levels and colorectal cancer in a prospective study. Int. J. Cancer 110: 617-620.

Cheng TC, Chen ST, Huang CS, Fu YP, et al. (2005). Breast cancer risk associated with genotype polymorphism of the catechol estrogen-metabolizing genes: a multigenic study on cancer susceptibility. Int. J. Cancer 113: 345-353.

Christman JK, Sheikhnejad G, Dizik M, Abileah S, et al. (1993). Reversibility of changes in nucleic acid methylation and gene expression induced in rat liver by severe dietary methyl deficiency. Carcinogenesis 14: 551-557.

Clemons M and Goss P (2001). Estrogen and the risk of breast cancer. N. Engl. J. Med. 344: 276-285.

Cochran WG (1950). The comparison of percentages in matched samples. Biometrika 37: 256-266.

Curtin K, Ulrich CM, Samowitz WS, Wolff RK, et al. (2011). Candidate pathway polymorphisms in one-carbon metabolism and risk of rectal tumor mutations. Int. J. Mol. Epidemiol. Genet. 2: 1-8.

Duval S and Tweedie R (2000). Trim and fill: A simple funnel-plot-based method of testing and adjusting for publication bias in meta-analysis. Biometrics 56: 455-463.

Egger M, Davey Smith G, Schneider M and Minder C (1997). Bias in meta-analysis detected by a simple, graphical test. BMJ 315: 629-634.

Foulkes WD (2008). Inherited susceptibility to common cancers. N. Engl. J. Med. 359: 2143-2153.

Fu TF, Hunt S, Schirch V, Safo MK, et al. (2005). Properties of human and rabbit cytosolic serine hydroxymethyltransferase are changed by single nucleotide polymorphic mutations. Arch. Biochem. Biophys. 442: 92-101.

Guerreiro CS, Carmona B, Goncalves S, Carolino E, et al. (2008). Risk of colorectal cancer associated with the C677T polymorphism in 5,10-methylenetetrahydrofolate reductase in Portuguese patients depends on the intake of methyldonor nutrients. Am. J. Clin. Nutr. 88: 1413-1418.

Hazra A, Wu K, Kraft P, Fuchs CS, et al. (2007). Twenty-four non-synonymous polymorphisms in the one-carbon metabolic pathway and risk of colorectal adenoma in the Nurses' Health study. Carcinogenesis 28: 1510-1519.

Heil SG, Van der Put NM, Waas ET, den Heijer M, et al. (2001). Is mutated serine hydroxymethyltransferase (SHMT) involved in the etiology of neural tube defects? Mol. Genet. Metab. 73: 164-172.

Higgins JP, Thompson SG, Deeks JJ and Altman DG (2003). Measuring inconsistency in meta-analyses. BMJ 327: 557560.

Hirschhorn JN, Lohmueller K, Byrne E and Hirschhorn K (2002). A comprehensive review of genetic association studies. Genet. Med. 4: 45-61. 
Komlosi V, Hitre E, Pap E, Adleff V, et al. (2010). SHMT1 1420 and MTHFR 677 variants are associated with rectal but not colon cancer. BMC Cancer 10: 525.

Koushik A, Kraft P, Fuchs CS, Hankinson SE, et al. (2006). Nonsynonymous polymorphisms in genes in the one-carbon metabolism pathway and associations with colorectal cancer. Cancer Epidemiol. Biomarkers Prev. 15: 2408-2417.

Lissowska J, Gaudet MM, Brinton LA, Chanock AJ, et al. (2007). Genetic polymorphisms in the one-carbon metabolism pathway and breast cancer risk: a population-based case-control study and meta-analyses. Int. J. Cancer 120: 26962703.

Melnyk S, Pogribna M, Miller BJ, Basnakian AG, et al. (1999). Uracil misincorporation, DNA strand breaks, and gene amplification are associated with tumorigenic cell transformation in folate deficient/repleted Chinese hamster ovary cells. Cancer Lett. 146: 35-44.

Mohammad NS, Yedluri R, Addepalli P, Gottumukkala SR, et al. (2011). Aberrations in one-carbon metabolism induce oxidative DNA damage in sporadic breast cancer. Mol. Cell. Biochem. 349: 159-167.

Moore LE, Malats N, Rothman N, Real FX, et al. (2007). Polymorphisms in one-carbon metabolism and trans-sulfuration pathway genes and susceptibility to bladder cancer. Int. J. Cancer 120: 2452-2458.

Naushad SM, Pavani A, Digumarti RR, Gottumukkala SR, et al. (2011). Epistatic interactions between loci of one-carbon metabolism modulate susceptibility to breast cancer. Mol. Biol. Rep. 38: 4893-4901.

Naushad SM, Pavani A, Rupasree Y, Divyya S, et al. (2012). Association of aberrations in one-carbon metabolism with molecular phenotype and grade of breast cancer. Mol. Carcinog. 51: E32-41.

Niclot S, Pruvot Q, Besson C, Savoy D, et al. (2006). Implication of the folate-methionine metabolism pathways in susceptibility to follicular lymphomas. Blood 108: 278-285.

Pharoah PD, Dunning AM, Ponder BA and Easton DF (2004). Association studies for finding cancer-susceptibility genetic variants. Nat. Rev. Cancer 4: 850-860.

Siegel R, Ma J, Zou Z and Jemal A (2014). Cancer statistics, 2014. CA Cancer J. Clin. 64: 9-29.

Steck SE, Keku T, Butler LM, Galanko J, et al. (2008). Polymorphisms in methionine synthase, methionine synthase reductase and serine hydroxymethyltransferase, folate and alcohol intake, and colon cancer risk. J. Nutrigenet. Nutrigenomics 1: 196-204.

Stevens VL, Rodriguez C, Sun J, Talbot JT, et al. (2008). No association of single nucleotide polymorphisms in onecarbon metabolism genes with prostate cancer risk. Cancer Epidemiol. Biomarkers Prev. 17: 3612-3614.

Succi M, de Castro TB, Galbiatti AL, Arantes LM, et al. (2014). DNMT3B C46359T and SHMT1 C1420T polymorphisms in the folate pathway in carcinogenesis of head and neck. Mol. Biol. Rep. 41: 581-589.

Vainer AS, Boiarskikh UA, Voronina EN, Selezneva IA, et al. (2010). Polymorphic variants of folate metabolizing genes (C677T and A1298C MTHFR, C1420T SHMT1 and G1958A MTHFD) are not associated with the risk of breast cancer in West Siberian Region of Russia. Mol. Biol. 44: 816-823.

van den Donk M, Visker MH, Harryvan JL, Kok FJ, et al. (2007). Dietary intake of B-vitamins, polymorphisms in thymidylate synthase and serine hydroxymethyltransferase 1, and colorectal adenoma risk: a Dutch case-control study. Cancer Lett. 250: 146-153.

Wang L, Lu J, An J, Shi Q, et al. (2007). Polymorphisms of cytosolic serine hydroxymethyltransferase and risk of lung cancer: a case-control analysis. Lung Cancer 57: 143-151.

Weiner AS, Oskina NA, Lacarev AF, Petrova VD, et al. (2012). Role of polymorphic variants of MTR gene A2756G and SHMT1 gene C1420T in the development of prostatic cancer in residents of the Western Siberian Region of Russia. Bull. Exp. Biol. Med. 152: 466-469.

Wu X, Zou T, Cao N, Ni J, et al. (2014). Plasma homocysteine levels and genetic polymorphisms in folate metablism are associated with breast cancer risk in chinese women. Hered. Cancer Clin. Pract. 12: 2.

Xu X, Gammon MD, Zhang H, Wetmur JG, et al. (2007). Polymorphisms of one-carbon-metabolizing genes and risk of breast cancer in a population-based study. Carcinogenesis 28: 1504-1509.

Yager JD (2000). Endogenous estrogens as carcinogens through metabolic activation. J. Natl. Cancer Inst. Monogr. 2000:67-73.

Yager JD and Liehr JG (1996). Molecular mechanisms of estrogen carcinogenesis. Annu. Rev. Pharmacol. Toxicol. 36: 203-232.

Yu CP, Wu MH, Chou YC, Yang T, et al. (2007). Breast cancer risk associated with multigenotypic polymorphisms in folate-metabolizing genes: a nested case-control study in Taiwan. Anticancer Res. 27: 1727-1732.

Zhang Z, Shi Q, Sturgis EM, Spitz MR, et al. (2005). Polymorphisms and haplotypes of serine hydroxymethyltransferase and risk of squamous cell carcinoma of the head and neck: a case-control analysis. Pharmacogenet. Genomics 15: 557-564.

Genetics and Molecular Research 14 (2): $5602-5614$ (2015) 